\title{
Proteasome inhibitors restore the STAT1 pathway and enhance the expression of MHC class I on human colon cancer cells
}

\author{
Yi-Hsin Liang 1,3,4,6, Kuo-Hsing Chen 1,4,6, Jia-Huei Tsai ${ }^{5}$, Yung-Ming Cheng ${ }^{5}$, Chang-Cheng Lee ${ }^{1,2}$, \\ Chiu-Hwa Kao ${ }^{1,2}$, Kuang-Yu Chan' ${ }^{1}$, Yeh-Ting Chen ${ }^{3}$, Wen-Ling Hsu ${ }^{3}$ and Kun-Huei Yeh 1,2,4,6*0
}

\begin{abstract}
Background: A new strategy, particularly a novel combination, for immunotherapy in microsatellite stable metastatic

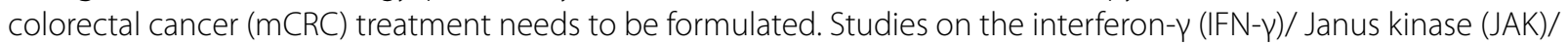
signal transducer and activator of transcription (STAT)1 pathway provide new directions in this regard.

Methods: Our study applies three colon cancer cell lines, including microsatellite stable (MSS) cell lines, which are SW480 and SW620, and microsatellite instability-high (MSI-H) cell line, which is DLD-1. We compared the expressions of immune surface markers on colon cancer cells in response to IFN- $\gamma$. We elucidated these mechanisms, which involved the upregulation of immune surface markers. Furthermore, we examined real-world clinical samples using the PerkinElmer Opal multiplex system and NanoString analysis.
\end{abstract}

Results: We established that the baseline expression of major histocompatibility complex (MHC) class I alleles and programmed death-ligand 1 (PD-L1) were generally low in cell line models. The immune surface markers were significantly increased after IFN- $\gamma$ stimulation on SW480 but were notably unresponsive on the SW620 cell line. We discovered that STAT1 and phosphorylated STAT1 (pSTAT1) were downregulated in the SW620 cell line. We verified that the STAT1/pSTAT1 could be restored through the application of proteasome inhibitors, especially bortezomib. The expression of MHC class I as downstream signals of STAT1 was also up-regulated by proteasome inhibitors. The similar results were reproduced in DLD-1 cell line, which was also initially unresponsive to IFN- $\gamma$. In real-world samples of patients with $\mathrm{mCRC}$, we found that higher STAT1 expression in tumor cells was strongly indicative of a highly immunogenic microenvironment, with significantly higher expression levels of MHC class I and PD-L1, not only on tumor cells but also on non-tumor cells. Furthermore, tumor infiltrating lymphocytes (TILs) were increased in the positive-STAT1 group. Through NanoString analysis, we confirmed that the mRNA expressions of IFN- $y$, human leukocyte antigen (HLA)-A, HLA-E, and HLA-G were also significantly higher in the positive-STAT1 group than those in the negative-STAT1 group.

Conclusion: Our study provides a novel rationale for the addition of bortezomib, a proteasome inhibitor, into new immunotherapy combinations.

Keywords: Colorectal cancer, Major histocompatibility complex (MHC) class I, Proteasome inhibitors, STAT1, Tumor infiltrating lymphocytes (TILs), Interferon- $\gamma($ IFN- $\gamma$ )

\footnotetext{
*Correspondence: khyeh@ntu.edu.tw

${ }^{1}$ Graduate Institutes of Oncology, National Taiwan University College of Medicine, No 7, Chung-Shan South Rd, Taipei 10002, Taiwan, R.O.C.

Full list of author information is available at the end of the article
}

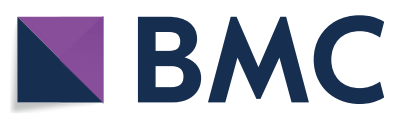

(c) The Author(s) 2021. Open Access This article is licensed under a Creative Commons Attribution 4.0 International License, which permits use, sharing, adaptation, distribution and reproduction in any medium or format, as long as you give appropriate credit to the original author(s) and the source, provide a link to the Creative Commons licence, and indicate if changes were made. The images or other third party material in this article are included in the article's Creative Commons licence, unless indicated otherwise in a credit line to the material. If material is not included in the article's Creative Commons licence and your intended use is not permitted by statutory regulation or exceeds the permitted use, you will need to obtain permission directly from the copyright holder. To view a copy of this licence, visit http://creativecommons.org/licenses/by/4.0/. The Creative Commons Public Domain Dedication waiver (http://creativeco mmons.org/publicdomain/zero/1.0/) applies to the data made available in this article, unless otherwise stated in a credit line to the data. 


\section{Background}

The development of immune checkpoint inhibitors has undergone considerable progress for patients with microsatellite instability-high (MSI-H) metastatic colorectal cancer (mCRC), which only comprises $1.8 \%-4.0 \%$ of all mCRC patients [1]. Chemotherapy and targeted therapy remain the primary treatment for more than $96 \%$ of patients with mCRC with microsatellite stable (MSS) tumors [2]. Various combination strategies have been established to treat this common form of mCRC; however, two phase III randomized trials provided disappointing results. In the IMblaze 370 study, applying atezolizumab with or without cobimetinib failed to exhibit greater overall survival than applying regorafenib [3]. In the MODUL study cohort-2, maintenance bevacizumab plus 5-fluorouracil (5-FU) with atezolizumab after first-line bevacizumab plus oxaliplatin and 5-FU treatment also failed to exhibit greater progression-free survival than maintenance bevacizumab plus 5-FU alone [4]. Novel strategies, especially combination strategies, for immunotherapy in mCRC with MSS tumors, are crucial for current researches.

Some biomarkers have been identified as predictors for immunotherapy, with the most critical biomarker being the interferon- $\gamma(\mathrm{IFN}-\gamma) /$ Janus kinase (JAK)/signal transducer and activator of transcription (STAT) 1 pathway [5, 6]. The IFN- $\gamma /$ JAK/STAT1 pathway plays a crucial role in the antigen processing pathway and the subsequent dynamic change of downstream signals, including major histocompatibility complex (MHC) class I [7-12]. STAT1 has also been reported to be a favorable prognostic factor for early-stage colorectal cancer (CRC) [13]. The ratio of STAT1 expression to STAT3 expression is a determinant of CRC growth [14]. In animal models, loss of STAT1 expression promotes the development of CRC $[15,16]$. However, the role of the IFN- $\gamma /$ JAK/STAT1 pathway in $\mathrm{mCRC}$ has received little attention [17, 18]. Although IFN- $\gamma$ also stimulates programmed cell death-ligand 1 (PD-L1) expression, IFN- $\gamma$ is not a standard treatment for mCRC [19]. Identification of the detailed interactions between STAT1 and PD-L1 might provide new insights for immunotherapy in mCRC.

In previous study, we have focused on the dynamic change of MHC class I in response to chemotherapy agents for mCRC [20]. We demonstrated that the higher expression of MHC class I on colon cancer cells implied higher vulnerability to immune surveillance. In the present study, we attempted to examine the expression of immune surface markers in colon cancer cells and elucidate the mechanisms underlying the effects of STAT1 on immune surface markers. Firstly, we applied our research on MSS colon cancer cell lines, which were SW480 and SW620 [21]. Then we reproduced our results on the
MSI-H colon cancer cell line, DLD-1 [21]. Although the correlation between proteasome and colon cancer were studied earlier [22, 23], the detailed interactions between proteasome and STAT1 for mCRC was not addressed before, and thus we would emphasize our research on this mechanism. Further, we also examined the correlations between the IFN- $\gamma /$ JAK/STAT1 pathway and other clinical characteristics with real-world samples from patients with mCRC.

\section{Methods \\ Cell lines}

We employed three colon cancer cell lines, SW480, SW620, and DLD-1 in our study. Both SW480 and SW620 were MSS, while the DLD-1 was MSI-H cell line [21]. All cell lines were purchased from the American Type Culture Collection. The cells were planted in T75 culture flasks (Thermo Fisher Scientific, 156499, Waltham, MA, USA) and maintained in RPMI-1640 medium (Gibco, 31800-022, Waltham, MA, USA) supplemented with 10\% FBS (Gibco, 10437-028) and 1\% amphotericin B, penicillin, and streptomycin (antibioticantimycotic; Gibco, 15240-062) in an atmosphere of 5\% $\mathrm{CO}_{2}$ at $37^{\circ} \mathrm{C}$.

\section{Chemicals and other reagents}

The compounds applied in this study for colon cancer cell lines were bortezomib (Cayman Chemical, 10008822, Ann Arbor, MI, USA), N-acetyl-L-leucyl-L-leucyl-Lnorleucinal (LLnL, Sigma-Aldrich, 208719, Darmstadt, Germany), MG132 (Sigma-Aldrich, 474790), and IFN- $\gamma$ (R\&D Systems, 285-IF-100, Minneapolis, MN, USA).

The chemicals applied for this experiment were xylene (J.T. Baker, JT-9490-03, Radnor, PA, USA), ethanol (Sigma-Aldrich, 32221), formaldehyde solution (10\% in aqueous phosphate buffer; Macron Fine Chemicals, H121-08, Radnor, PA, USA), 10× TBS pH 7.4 (Protech, BF204, Taipei, Taiwan, R.O.C), TWEEN 20 (MyBioSource, MBS4156394, San Diego, CA, USA), 10× EDTA buffer pH 8.5 (Sigma-Aldrich, E1161), 10× citrate buffer pH 6.0 (Sigma-Aldrich, C9999), and ProLong Diamond Antifade Mountant (Thermo Fisher Scientific, P36965).

\section{Flow cytometry}

The antibodies applied for flow cytometry were phycoerythrin (PE) anti-human pan-MHC class I (BioLegend, 311406, San Diego, CA, USA), purified anti-human HLA-A (HLA-A; MyBioSource, MBS438658), PE anti-mouse immunoglobulin G1 (IgG1; BioLegend 406608), purified anti-human HLA-C (HLA-C; BioLegend, 373302), PE anti-mouse IgG2b (IgG2b; BioLegend, 406708), PE anti-human HLA-E (HLA-E; BioLegend, 342604), PE anti-human HLA-F (HLA-F; BioLegend, 
373204), PE anti-human HLA-G (HLA-G; BioLegend, 335906), PE anti-human CD274 (B7-H1, PD-L1, BioLegend, 329706), PE-IgG1 isotype control (BioLegend, 400112), PE IgG2a isotype control (BioLegend, 400212), and PE-IgG2b isotype control (BioLegend, 402204).

The flow cytometry procedures were described in the earlier study [20]. Briefly summarized, we seeded $10^{6}$ cells with $10-\mathrm{mL}$ culture medium in $10-\mathrm{cm}$ dishes for $24 \mathrm{~h}$. These cells were treated with IFN- $\gamma 100 \mathrm{U} / \mathrm{mL}$ for the subsequent $24 \mathrm{~h}$ and then were harvested with trypsin (Gibco, 15400-054) and centrifuged with $500 \mathrm{~g}$ for $5 \mathrm{~min}$. In each eppendorf tube, $5 \times 10^{5}$ cells were infused with $200 \mu \mathrm{L}$ cold phosphate-buffered saline (PBS; Gibco, 10010-023) and recentrifuged. After removing the supernatant, $5 \mu \mathrm{L}$ of fluorescent antibody (including isotype control antibodies) with $50 \mu \mathrm{L}$ cold PBS (antibody: cold $\mathrm{PBS}=1: 10$ ) were applied and the samples were put on ice and in darkness for $20 \mathrm{~min}$. After centrifuging with $500 \mathrm{~g}$ for $5 \mathrm{~min}$ for twice, the cells were analyzed by flow cytometer (BD FACSCalibur; BD Biosciences, Franklin Lakes, NJ, USA).

\section{Western blot}

We applied enhanced chemiluminescence Immobilon Crescendo Western horseradish peroxidase (HRP) substrate (Merck Millipore, WBLUR0500, Darmstadt, Germany) for these Western blots. The antibodies applied for Western blots were STAT1 (Cell Signaling Technology, \#9172, Danvers, MA, USA), phosphorylated STAT1 (pSTAT1; Cell Signaling Technology, \#9167), phospho-JAK1 (pJAK1; Cell Signaling Technology, \#3331), JAK1 (Cell Signaling Technology, \#3332), phosphoJAK2 (pJAK2; Cell Signaling Technology, \#3771), JAK2 (Cell Signaling Technology, \#3230), interferon regulatory factor-1 (IRF-1; Cell Signaling Technology, \#8478), pan-MHC class I (Origene, AM33035PU-N, Rockville, MD, USA), ß-actin (Abcam, ab8227, Cambridge, UK), GAPDH (Abcam, ab8245), HRP donkey anti-rabbit IgG (BioLegend, 406401), and HRP goat anti-mouse IgG (BioLegend, 405306).

The Western blot procedures were described amply in the earlier study [20]. Briefly summarized, protein concentrations were measured using the bicinchoninic acid assay. Then the electrophoresis was applied in $10 \%$ sodium dodecyl sulfate polyacrylamide gel electrophoresis (SDS-PAGE, $15 \mu \mathrm{g}$ per lane) and transferred onto polyvinylidene fluoride blotting membranes (Merck Millipore, IPVH00010). The membranes were incubated in $5 \%$ nonfat milk in TBS containing 0.1\% TWEEN 20 for blockage of nonspecific binding sites for $1 \mathrm{~h}$, and the membranes were incubated overnight at $4{ }^{\circ} \mathrm{C}$ with primary antibodies. On the next day, the membranes were washed with TBS containing 0.1\% TWEEN 20 before and after incubation with HRP-conjugated secondary antibodies. These membranes were then incubated with enhanced chemiluminescence Western blotting detection reagents (Merck Millipore, WBLUR0500, Darmstadt, Germany) and exposed to film.

\section{Patients and clinical samples}

We enrolled patients at National Taiwan University Hospital with the following eligibility criteria: (1) patients aged $\geq 20$ years; (2) patients with metastatic CRC; and (3) patients without known history of receiving immune modification drugs, such as steroids, immunosuppressive agents, and immunotherapy under clinical trial settings. Informed consents were obtained at diagnosis of metastatic disease status. Primary tumor tissues, including archival tissues or fresh biopsies, from these participants were obtained for subsequent analysis after receiving informed consents.

\section{Immunohistochemical staining of patient samples}

The IHC staining procedures were detailed in the earlier study [20]. Briefly summarized, we applied the Opal 7 Solid Tumor Immunology Kit (PerkinElmer, OP7TL4001KT) for multiplex IHC staining. The PerkinElmer Opal multiplex system could simultaneously apply up to 7 different IHC staining for each cell. The 4',6-diamidino-2-phenylindole (DAPI) was applied for identification of nucleus. The pan-cytokeratin (pan-CK) was applied for identification of CRC cells. The rest of five biomarkers were applied for identification of immune microenvironment. The antibodies applied for immunohistochemical (IHC) staining were STAT1 (Abcam, ab31369), PD-L1 (Cell Signaling Technology, \#13684), MHC class I (Abcam, ab134189), CD4 (PerkinElmer, OP7TL4001KT), CD8 (PerkinElmer, OP7TL4001KT), and pan-CK (Abcam, ab7753). We applied EDTA buffer pH 8.5 (Sigma-Aldrich, E1161), citrate buffer pH6.0 (Sigma-Aldrich, C9999), antibody diluent (PerkinElmer, ARD1001EA, Waltham, MA, USA), Opal Polymer HRP secondary antibody solution (PerkinElmer, ARH1001EA), Opal fluorophore solution (PerkinElmer, FP1487001KT), DAPI solution (PerkinElmer, FP1490A), and ProLong Diamond Antifade Mountant (Thermo Fisher Scientific, P36965) in our study. Quantification of the biomarkers in the tissue sections were performed using the Vectra Polaris Automated Quantitative Pathology Imaging System along with inForm analysis software (PerkinElmer, CLS143455). Detailed information is available on the official website at https://www.perkinelmer.com/lab-solut ions/resources/docs/DTS_1-05-40-NR-OPALGUIDEL INES_Opal4-7-color_Manual_Kit_Insert.pdf 


\section{NanoString assay of patient samples}

We applied a commercial multiplexed gene expression panel, the NanoString nCounter PanCancer Immune Profiling Panel (NanoString Technologies, Seattle, WA, USA). The NanoString panel can simultaneously quantitate 770 immune-related genes through mRNA quantification. All procedures, including preparation, hybridization, detection, scanning, and normalization, were performed according to the manufacturer's instructions. Detailed information and the gene list are available on the official website at https://www.nanos tring.com/products/gene-expression-panels/gene-expre ssion-panels-overview/hallmarks-cancer-gene-expre ssion-panel-collection/pancancer-immune-profilingpanel. Briefly summarized, we obtained $12-15$ slides of $10 \mu \mathrm{m}$-thickness from formalin-fixed paraffin-embedded specimens. After referring to the representative hematoxylin and eosin slide, viable tumor portion was microdissected from the other unstained slides. Total 200-500 ng RNA was extracted and the percentage of RNA fragments greater than 300 nucleotides (DV300) was greater than 20\%. After hybridization with the Immune Profiling Panel by the NanoString Prep Station (NanoString Technologies), sample analysis was performed on a nCounter Digital Analyzer (NanoString Technologies). The raw data processing, quality control, and normalization were performed using the nSolver 4.0 analysis software (NanoString Technologies) after reference gene normalization with 40 house-keeping genes.

\section{Statistical analysis}

All results, except for clinical samples, are based on at least three independent tests. The results are presented in terms of the mean with standard deviation, and are graphed. We applied ImageJ (RRID:SCR_003070, National Institutes of Health, Bethesda, MD, USA) to quantify the results of Western blots. We performed a paired samples $t$ test or ANOVA as indicated. All $p$ values were two-tailed. The $p$ values are marked with asterisks to indicate their level of significance. All data analyses were performed using SPSS 20.0 (RRID:SCR_002865, IBM, Armonk, NY, USA).

\section{Results}

IFN- $\gamma$ specifically stimulated the expression of stimulatory MHC class I alleles and PD-L1 in SW480 but not in SW620 cells. First, we examined two colon cancer cell lines, SW480 and SW620, through flow cytometry. The SW480 line demonstrated low expression of all MHC class I alleles and PD-L1 at baseline (Fig. 1A). IFN- $\gamma$ significantly stimulated the expression of PD-L1 and MHC class I alleles except for HLA-F. In contrast, the SW620 line was completely unresponsive to IFN- $\gamma$ stimulation for all MHC class I alleles and PD-L1 (Fig. 1B). To elucidate the differences between the SW480 and SW620 cell lines, we subsequently investigated the IFN- $\gamma$ pathway by using Western blot. After exposure to IFN- $\gamma$, the downstream signals of JAK1/JAK2, STAT1, and IRF1 increased in SW480 cells (Fig. 1C). By contrast, STAT1 and PSTAT1 were downregulated in the SW620 cell line.

Proteasome inhibitors restored the expression of pSTAT1 and downstream signals, including IRF1 and MHC class I in SW620 cell line. The proteasome-ubiquitin mechanism is crucial for the modulation of immune systems [24]. Thus, we examined three proteasome inhibitors, namely bortezomib, LLnL, and MG132. All three proteasome inhibitors restored the levels of STAT1 and pSTAT1. The downstream signal of IRF1 was also upregulated (Fig. 2A \& B).

Bortezomib enhanced MHC class I expression in a dose-dependent manner in SW620 and DLD-1 cell lines. Because bortezomib is currently one of the standard treatments for hematological malignancies, we further examined its dosage efficacy in SW620 cell line $[25,26]$. The levels of pSTAT1 and IRF1 increased with the dosage of bortezomib. The expression of MHC class I was stimulated by the application of bortezomib in a dose-dependent manner (Fig. 3A \& B). To reproduce these results, we applied another cell line in our study. As shown in the Fig. 3C and D, the levels of pSTAT1 and IRF1 were restored with the dosage of bortezomib in the DLD-1 cell line. Again, the expression of MHC class I was also stimulated by the application of bortezomib in the DLD-1 cell line. In summary, proteasome might degrade STAT1 at baseline and thus tumor cells were unresponsive to IFN- $\gamma$ stimulation. The proteasome inhibitors restore the expression of STAT1 in SW620 and DLD-1 cell lines. The stimulation of IFN- $\gamma /$ JAK/STAT1 pathway subsequently upregulated the expressions of MHC class I (Fig. 3E).

$M H C$ class $I$ and $P D-L 1$ expression were strongly correlated with STAT1 expression in real-world patients' samples. We enrolled 60 patients with mCRC in this study. Their clinical characteristics and molecular biomarkers are summarized in Table 1 . Only 4 patients had MSI-H tumors and the rest of 56 patients had MSS tumors. All 60 tumor tissues from primary colorectal tumors before $\mathrm{mCRC}$ treatment were examined with the PerkinElmer Opal multiplex system, which combines seven different IHC staining signals for each cell within one image, as shown in Fig. 4A. The immune microenvironment was composed by tumor cells and peri-tumor non-tumor cells, including immune cells. As defined by previous studies, tumor cells were identified as both panCK-positive and DAPI-positive cells. Non-tumor cells infiltrated in the tumors, such as tumor infiltrating lymphocytes (TILs), were categorized as pan-CK negative 
A

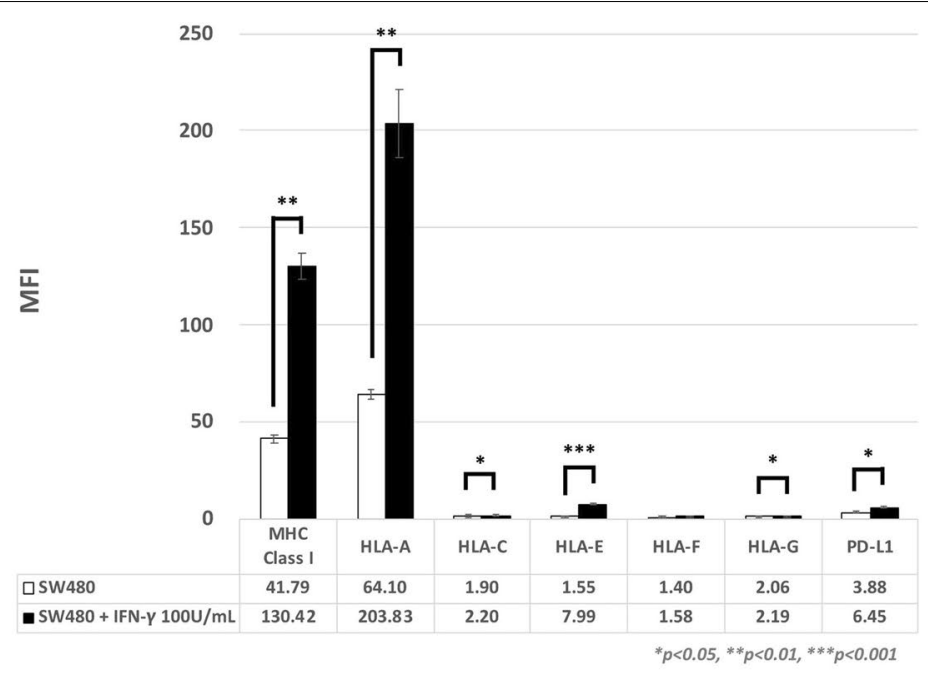

B

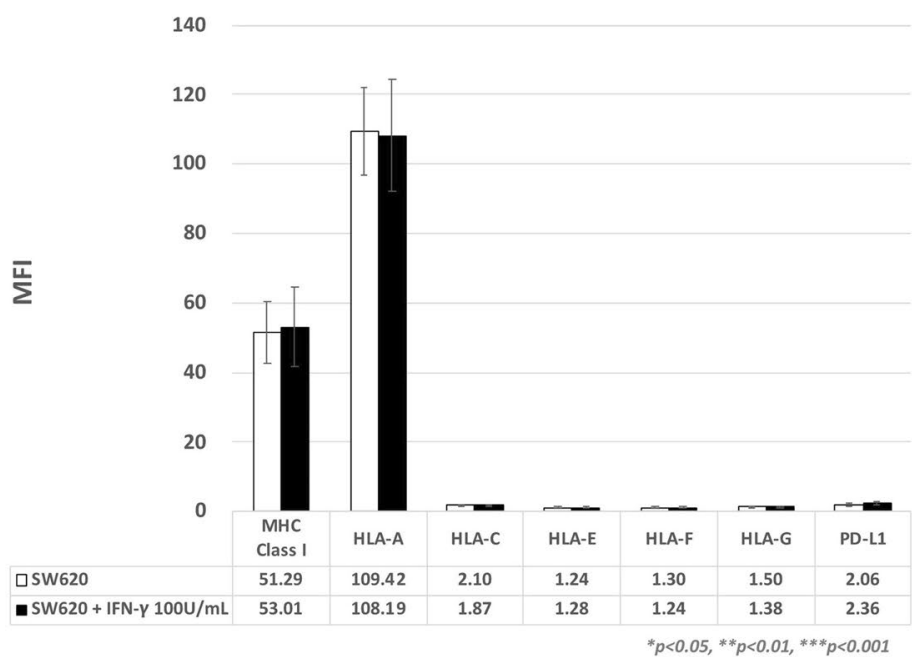

C

SW620

0 15' $30^{\prime} 1 \mathrm{~h} 2 \mathrm{~h}$

- - -

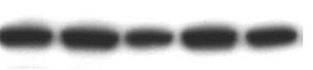

$---$
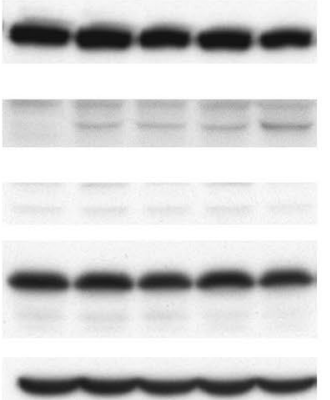

SW480

0 15' $30^{\prime} 1 \mathrm{~h} 2 \mathrm{~h}$

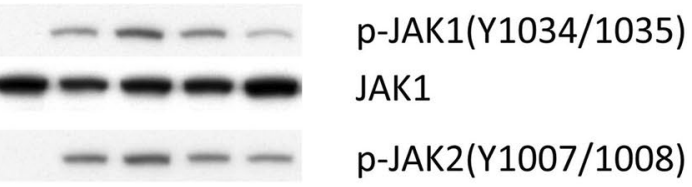

JAK2

p-STAT1(Y701)

\section{STAT1}

IRF-1

\section{ß-actin}

Fig. 1 Flow cytometry of SW480 (A) and SW620 (B) for MHC class I alleles and PD-L1 expressed in response to IFN- $\gamma$ after 24-h exposure. C Western blots for the SW480 and SW620 cells in the IFN- $\gamma$ pathway in response to IFN- $\gamma$ after 15 min (15'), 30 min $\left(30^{\prime}\right), 1 \mathrm{~h}(1 \mathrm{H})$, and $2 \mathrm{~h}(2 \mathrm{H})$. Mean fluorescence intensity (MFI) 


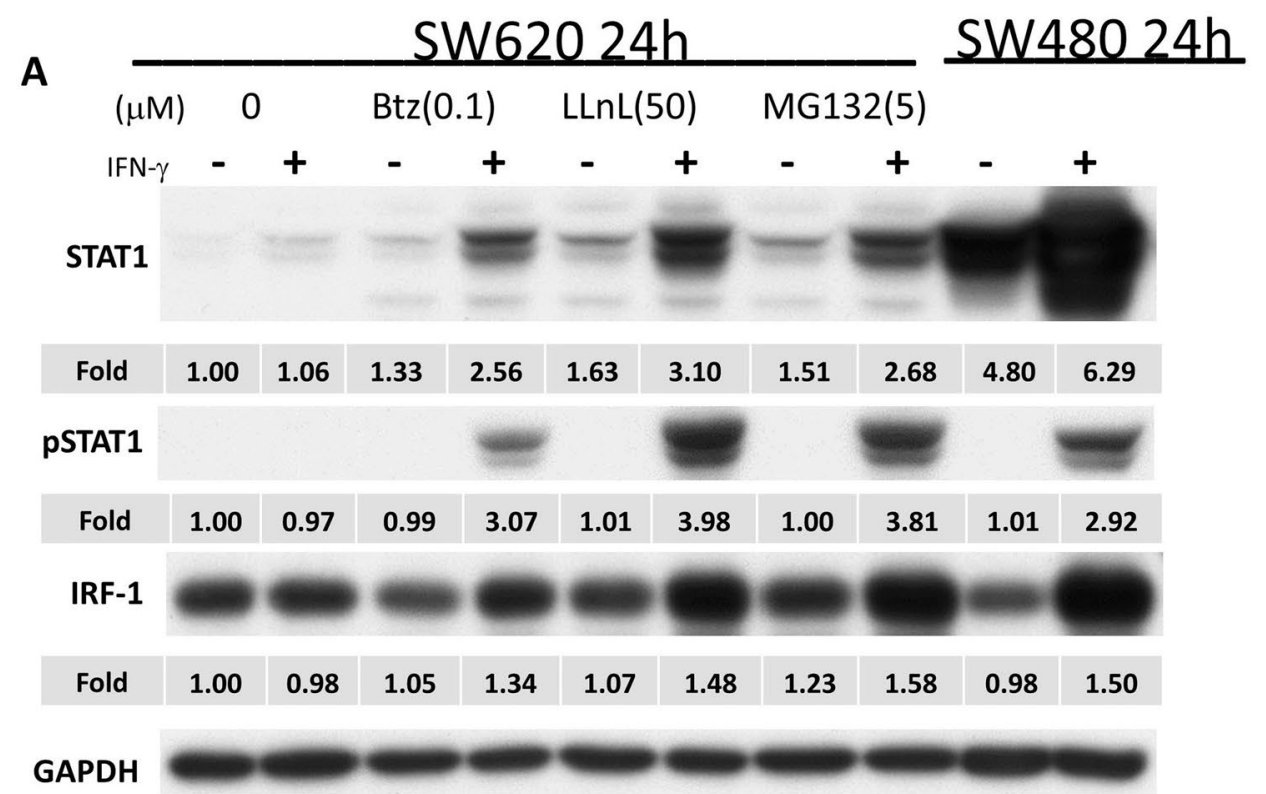

B
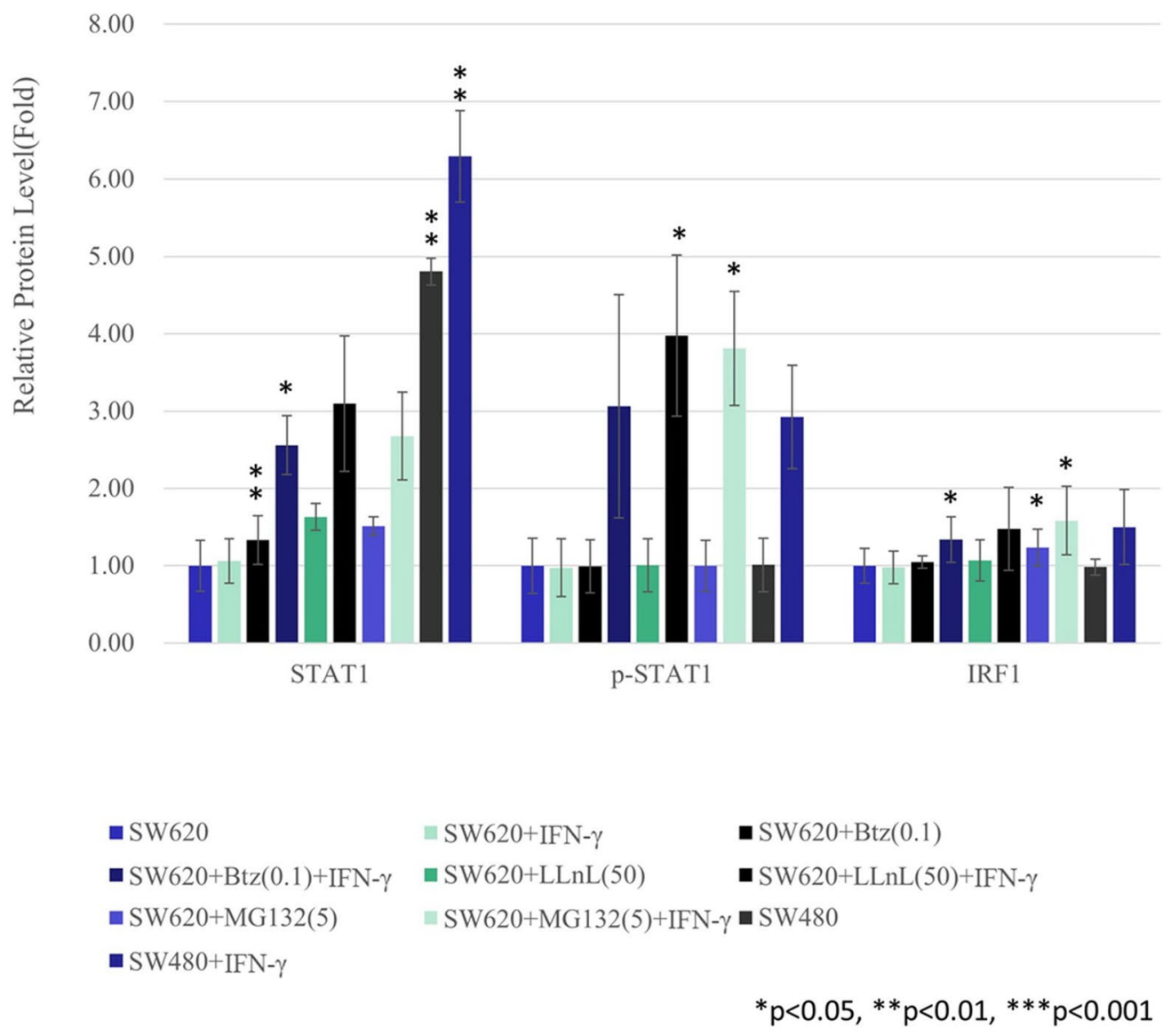

Fig. 2 Western blots (A) and the quantification of Western blots (B) for SW620 and SW480 cells in the STAT1 pathway in response to bortezomib, LLnL, MG132, and IFN- $\gamma$ 
A

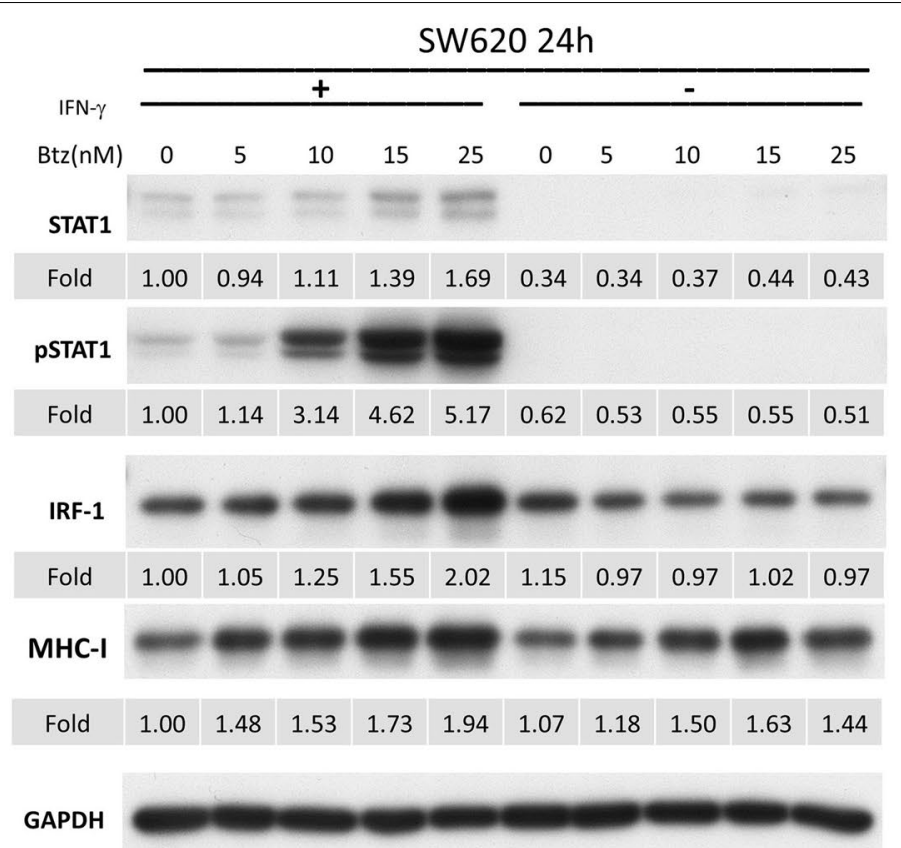

B

800

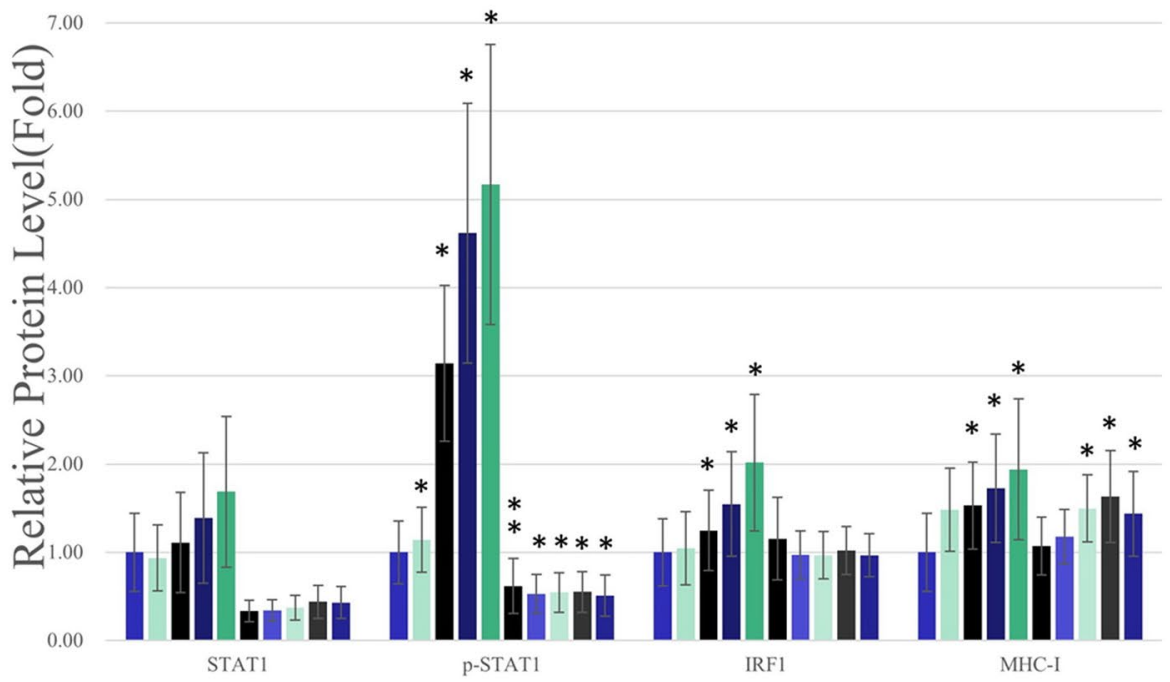

$\square \mathrm{SW} 620+\mathrm{IFN}-\gamma \quad$ Btz $5 \mathrm{nM}+\mathrm{IFN}-\gamma \quad \boldsymbol{\nabla} \mathrm{Btz} 10 \mathrm{nM}+\mathrm{IFN}-\gamma$

- Btz $15 \mathrm{nM}+\mathrm{IFN}-\gamma \quad$ Btz $25 \mathrm{nM}+\mathrm{IFN}-\gamma \quad$ a SW620

Btz $5 \mathrm{nM}$

Btz 10nM

Btz $15 \mathrm{nM}$

- Btz 25nM

Fig. 3 Western blots (A) and the quantification of western blots (B) for SW620 cells in the STAT1 pathway in response to bortezomib and IFN- $\gamma$. We further applied DLD-1 cell line with western blots $(\mathbf{C})$ and the quantification of western blots (D) for confirmation. Schema of the mechanism of proteasome inhibitors on MHC class I expression of colon cancer cell lines was summarized in Fig. 3 (E) 


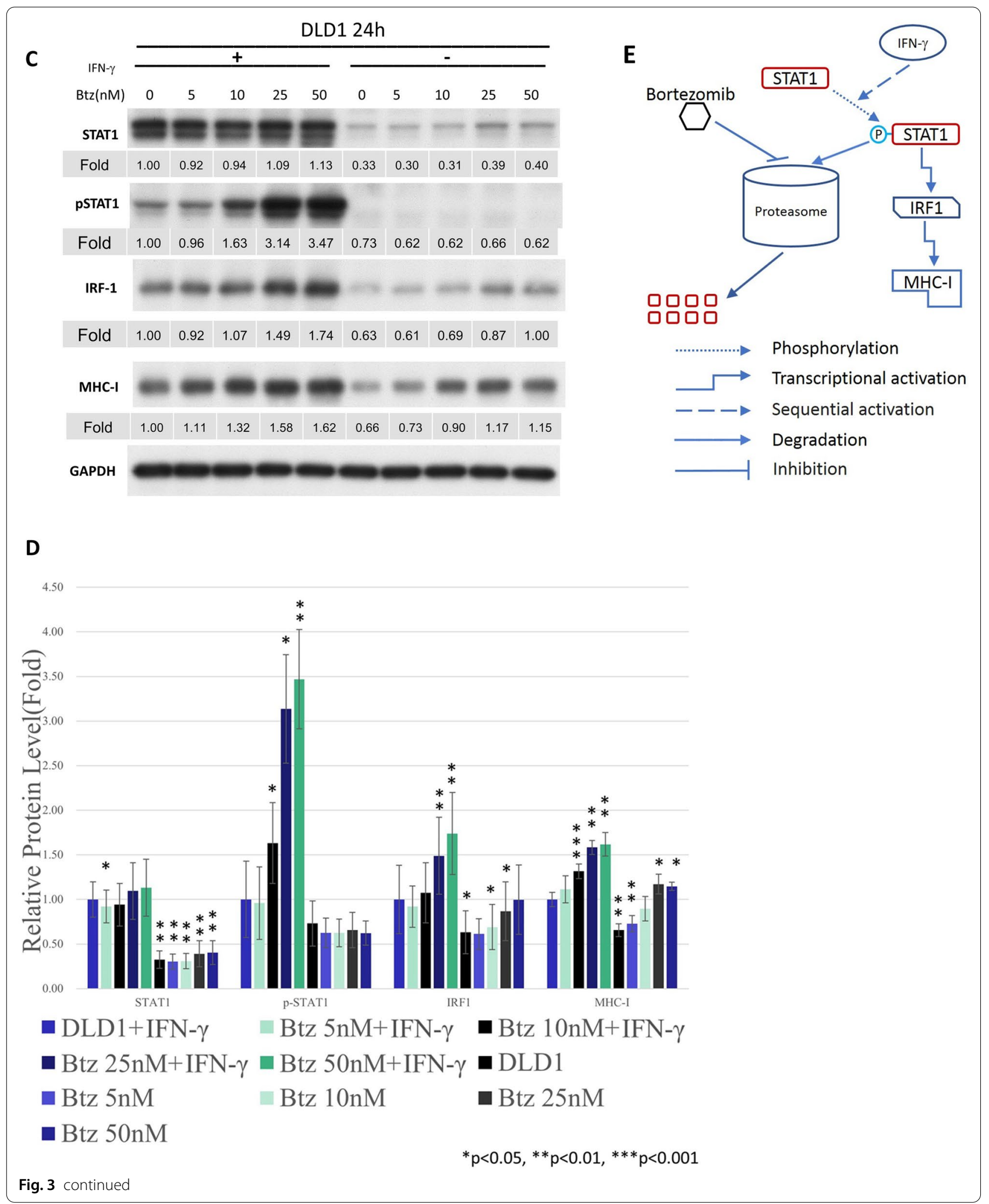


Table 1 Patient clinical characteristics

\begin{tabular}{|c|c|c|c|c|}
\hline Characteristic & All patients $(\%)^{a}$ & Tumor STAT1 $\geqq 1 \%(\%)^{\mathrm{a}}$ & Tumor STAT1 $<1 \%(\%)^{\mathrm{a}}$ & $p$-value \\
\hline All & $60(100 \%)$ & $32(53.3 \%)^{b}$ & $28(46.7 \%)^{b}$ & \\
\hline \multicolumn{5}{|l|}{ Gender } \\
\hline Female & $31(51.7 \%)$ & $16(50.0 \%)$ & $15(53.6 \%)$ & \multirow[t]{2}{*}{0.782} \\
\hline Male & $29(48.3 \%)$ & $16(50.0 \%)$ & $13(46.4 \%)$ & \\
\hline \multicolumn{5}{|l|}{ Age (years) } \\
\hline Median (Range) & $59.8(36-84)$ & $54.6(38-85)$ & $62.5(36-81)$ & \\
\hline Age $\leq 65$ years & 42 (70.0\%) & 23 (71.9\%) & 19 (67.9\%) & \multirow[t]{2}{*}{0.735} \\
\hline Age $>65$ years & $18(30.0 \%)$ & $9(28.1 \%)$ & $9(32.1 \%)$ & \\
\hline \multicolumn{5}{|l|}{ Primary site } \\
\hline Right-sided $^{c}$ & $22(36.7 \%)$ & $11(34.4 \%)$ & $11(39.3 \%)$ & \multirow[t]{2}{*}{0.694} \\
\hline Left-sided $^{d}$ & $38(63.3 \%)$ & $21(65.6 \%)$ & $17(60.7 \%)$ & \\
\hline \multicolumn{5}{|l|}{ Initial stage } \\
\hline |-III & $4(6.7 \%)$ & $4(12.5 \%)$ & $0(0 \%)$ & \multirow[t]{2}{*}{0.156} \\
\hline IV & $56(93.3 \%)$ & $28(87.5 \%)$ & $28(100 \%)$ & \\
\hline \multicolumn{5}{|l|}{ Mutation status } \\
\hline Wild type & $26(43.3 \%)$ & $16(50 \%)$ & $10(35.7 \%)$ & \multirow[t]{4}{*}{0.516} \\
\hline RAS mutant & $25(41.7 \%)$ & $12(37.5 \%)$ & $13(46.4 \%)$ & \\
\hline BRAF mutant & $6(10.0 \%)$ & $3(9.4 \%)$ & $3(10.7 \%)$ & \\
\hline $\mathrm{MSI}-\mathrm{H}$ & $4(6.7 \%)$ & $1(3.1 \%)$ & $3(10.7 \%)$ & \\
\hline \multicolumn{5}{|l|}{ Tumor PD-L1 } \\
\hline$<1 \%$ & $27(45.0 \%)$ & $7(21.9 \%)$ & $20(71.4 \%)$ & \multirow[t]{2}{*}{$<0.001^{\mathrm{e}}$} \\
\hline$\geqq 1 \%$ & $33(55.0 \%)$ & $25(78.1 \%)$ & $8(28.6 \%)$ & \\
\hline \multicolumn{5}{|l|}{ Non-tumor PD-L1 } \\
\hline$<1 \%$ & $48(80.0 \%)$ & $22(68.8 \%)$ & $26(92.9 \%)$ & \multirow[t]{2}{*}{$0.020^{e}$} \\
\hline$\geqq 1 \%$ & $12(20.0 \%)$ & $10(31.2 \%)$ & $2(7.1 \%)$ & \\
\hline \multicolumn{5}{|l|}{ Tumor MHC-1 } \\
\hline$<50 \%$ & $12(20.0 \%)$ & $0(0.0 \%)$ & $12(42.9 \%)$ & \multirow[t]{2}{*}{$<0.001^{\mathrm{e}}$} \\
\hline$\geqq 50 \%$ & $48(80.0 \%)$ & $32(100.0 \%)$ & $16(57.1 \%)$ & \\
\hline \multicolumn{5}{|l|}{ Non-tumor MHC-1 } \\
\hline$<50 \%$ & $20(33.3 \%)$ & $6(18.7 \%)$ & $14(50.0 \%)$ & \multirow[t]{2}{*}{$0.010^{e}$} \\
\hline$\geqq 50 \%$ & $40(66.7 \%)$ & $26(81.3 \%)$ & $14(50.0 \%)$ & \\
\hline \multicolumn{5}{|l|}{ Non-tumor CD4, CD8 } \\
\hline $\begin{array}{l}\text { Both }<1 \% \\
\text { CD4 or CD8 } \\
\geqq 1 \%\end{array}$ & $\begin{array}{l}34(56.7 \%) \\
26(43.3 \%)\end{array}$ & $\begin{array}{l}11(34.4 \%) \\
21(65.6 \%)\end{array}$ & $\begin{array}{r}23(82.1 \%) \\
5(17.9 \%)\end{array}$ & $<0.001^{e}$ \\
\hline
\end{tabular}

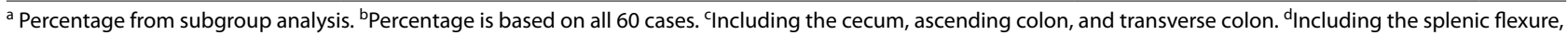
descending colon, sigmoid colon, and rectum. ${ }^{e} p<0.05$

and DAPI-positive cells [27, 28]. We applied another 5 biomarkers, which were STAT1, CD4, CD8, MHC class $\mathrm{I}$, and PD-L1, in reference with DAPI and CK to construct the immune microenvironment for each sample. Because the raw image data was too complicated to be visualized manually, we applied computerized scanning of each IHC staining for all 60 samples, and only demonstrated one patient sample as an example in Fig. 4BG. The computerized scanning processed the STAT1 (Fig. 4B), MHC class I (Fig. 4C), PD-L1 (Fig. 4D), CD4 (Fig. 4E), CD8 (Fig. 4F) respectively. These signals were combined in reference of DAPI and CK to identify the interaction of tumor cells and non-tumor cells by computer (Fig. 4G). As shown in Fig. 4G, the tumor parts were marked as brown background and the non-tumor parts were marked as green background. For example, we could identify the STAT1 expression and the MHC class I expression on each tumor cell and/or non-tumor cell in reference of the synchronous expression of DAPI and CK or not. These statistic results were summarized in Table 1 and more detailed results could be found in the Additional file 1. The definitions of positivity for these 

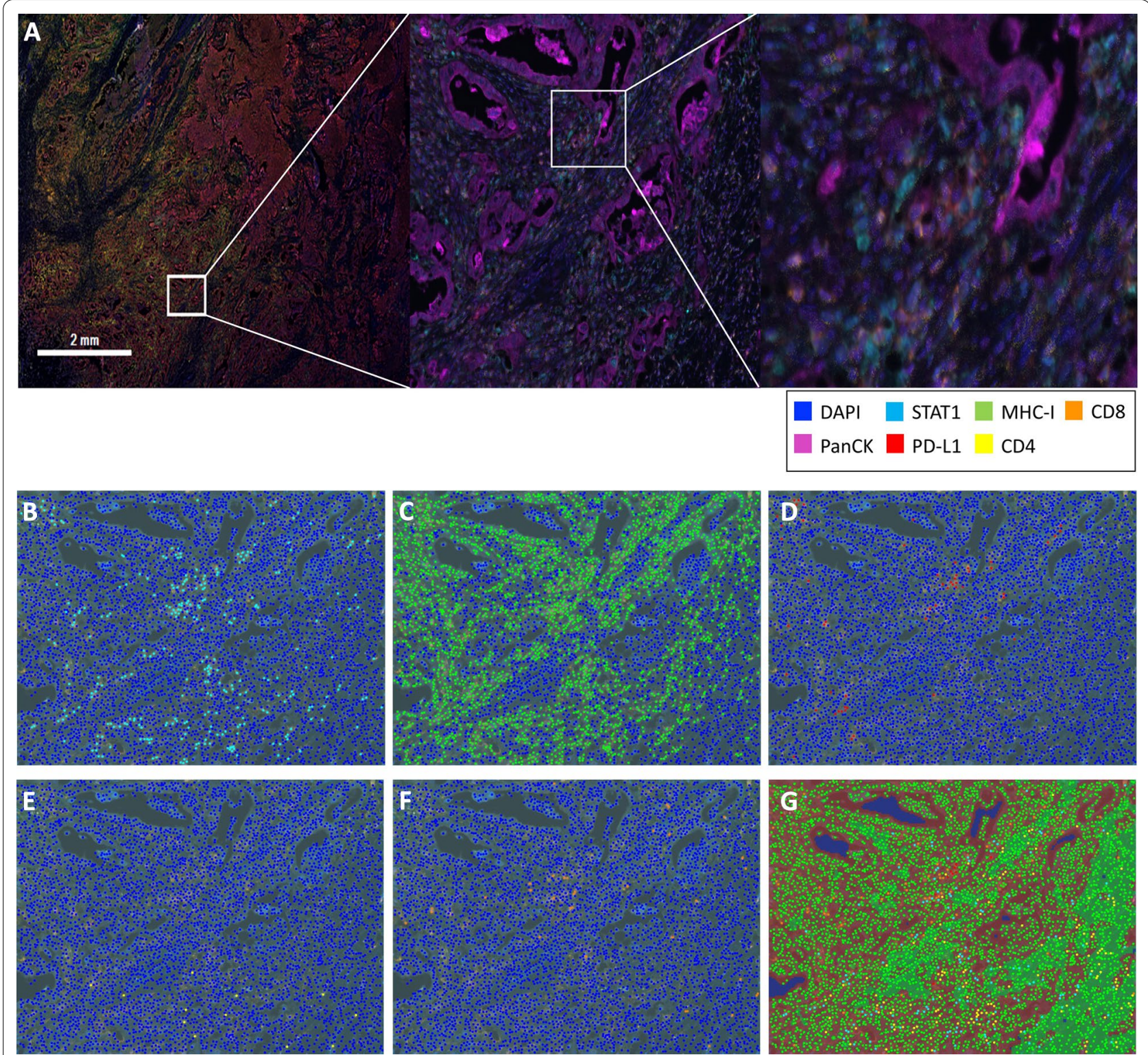

Fig. 4 One example of IHC staining by the PerkinElmer Opal multiplex system. The merge of all seven different IHC staining within one picture was demonstrated as (A). The individual computerized scanning of STAT1 IHC staining (B), MHC class I IHC staining (C), PD-L1 IHC staining (D), CD4 IHC staining $(\mathbf{E})$, and $\mathrm{CD} 8 \mathrm{IHC}$ staining $(\mathbf{F})$ were demonstrated. Computerized scanning of superimposing all five $\mathrm{IHC}$ staining was demonstrated in $(\mathbf{G})$

biomarkers were referenced by the definition of PD-L1. The definitions of positivity of STAT1, PD-L1, CD4, and CD8 were defined as positive expression on $\geqq 1 \%$ of tumor cells or non-tumor cells, respectively. The definition of positivity of MHC class I was defined as positive expression on $\geqq 50 \%$ of tumor cells or non-tumor cells. We observed that $32(53.3 \%)$ of 60 samples were STAT1 positive tumors. STAT1 expression was not correlated with other clinical factors, such as RAS and MSI-H status. However, a positive tumor STAT1 expression implied a strong immune microenvironment. The positive tumor
STAT1 expression was significantly associated with higher PD-L1 expression and higher MHC class I expression, on tumor cells and also non-tumor cells. The level of TILs also increased in the positive STAT1 group with significantly more cells expressed CD4 or CD8.

NanoString analysis indicated that MHC class I expression, especially that for $H L A-A$, and IFN- $\gamma$, significantly increased in real-world patients' samples. We further subjected these patients' tumors samples to NanoString analysis. Consistent with the grouping of results from the PerkinElmer Opal multiplex system, STAT1 expression 
was significantly higher in the positive-STAT1 group than in the negative-STAT1 group (Fig. 5A). The expressions of all specific MHC class I alleles were higher in the positive-STAT1 group, and the differences were especially significant for HLA-A, HLA-E, and HLA-G (Fig. 5B-F). The NanoString analysis also verified the reason that high STAT1 and MHC class I expression was strongly correlated with IFN- $\gamma$ expression. The IFN- $\gamma$ expression was significantly higher in the positive-STAT1 group than in the negative-STAT1 group (Fig. 5G).

\section{Discussion}

In this study, we demonstrated that colon cancer can be separated into two groups according to their response or lack of response to IFN- $\gamma$. We discovered that SW620 was irresponsive to IFN- $\gamma$ because of the downregulation of STAT1. We further found that bortezomib, a proteasome inhibitor, can restore STAT1 expression and subsequently enhance the downstream expression of $\mathrm{MHC}$ class I. The real-world mCRC samples were classified into two groups according to their STAT1 expression level. The positive STAT1 expression in mCRC cells was strongly indicative of a highly immunogenic microenvironment, with significantly higher expression levels of MHC class I and PD-L1, in both tumor and nontumor parts, compared with cells with negative STAT1 expression. STAT1 expression was an independent biomarker without any interaction with other molecular biomarkers, such as RAS or MSI-H mutations.

Currently, microsatellite stable mCRC is still the no man's land for immunotherapy. In previous study, we have demonstrated that the up-regulation of MHC class I made the colon cancer cells more vulnerable to immune surveillance [20]. In this study, we confirmed that the positive tumor STAT1 expression implied higher MHC class I expression and PD-L1 expression on tumor cells and also peri-tumor non-tumor cells, such as TILs. STAT1 expression levels of mCRC might be a predictive biomarker for novel immunotherapy. Furthermore, we have established that bortezomib is a candidate of immunotherapy combination for the negative STAT1 expression $\mathrm{mCRC}$.

Although our research indicated that the STAT1 pathway was independent to microsatellite status and could be applied for mCRC with either MSS tumors or MSI-H tumors, the impactful value of our results was addressed on the novel immunotherapy strategy for MSS mCRC. As mentioned above, the MSI-H mCRC comprised only small proportions of the whole $\mathrm{mCRC}$ and current immunotherapy provided perfect efficacy on MSI-H mCRC. On the contrary, MSS mCRC was resistant to immunotherapy. The STAT1 pathway provided a new biomarker and a potential mechanism of action for immunotherapy on MSS mCRC.

STAT1 plays a crucial role in determining the immune microenvironment in mCRC. STAT1 has a strong interaction with indoleamine-2,3-dioxygenase-1 expression in Paneth cells and with subsequent immune escape in CRC [29]. STAT1 play an important role in early tumorigenesis of CRC [30, 31]. Sakahara et al. demonstrated that the STAT1 might be correlated with the drug response [16]. Furthermore, IFN- $\gamma /$ JAK/STAT1 signaling stimulates PD-L1 expression in CRC cells [32]. Kikuchi et al. have indicated that the co-expression of PSTAT1 and PD-L1 in CRC cells was strongly correlated with CD4- and CD8positive TILs [33]. Our present study discovered the strong correlation with the IFN- $\gamma /$ JAK/STAT1 signaling and the major immunogenic action from the stimulation of MHC class I. By using PerkinElmer Opal multiplex system, we drew the same results that the higher expressions of STAT1 and MHC class I subsequently enhanced "hot" immunogenic microenvironment, which was recognized by significantly higher percentage of TILs. The upregulation of STAT1 implied a "hot" immunogenic microenvironment, which might indicate greater vulnerability to immunotherapy.

Several uncertainties remain regarding immunotherapy for microsatellite-stable mCRC. Various combination strategies, including the incorporation of standard chemotherapy or novel targeted therapy, have failed to deliver satisfactory results. A new pathway or novel combination is crucial for effective treatment. The proteasomeubiquitin pathway plays a major role in regulating STAT expression and thus represents a potential breakthrough [24]. Our research indicated that bortezomib, a proteasome inhibitor, restored MHC class I expression, and thus subsequently transformed the "cold" immune microenvironment into a "hot" microenvironment. Proteasome inhibitors are favorable candidates for further immunotherapy combinations.

The present study has some limitations. First, our data could be applied on STAT1-related mCRC but not all mCRC subtypes. $\mathrm{mCRC}$ is a heterogeneous entity that has been classified into at least four specific molecular subtypes [34, 35]. More novel treatments are required for effective treatment in various immune microenvironments. Second, the stimulation of MHC class I might only be the first step in effective treatment. Our study simplified the role of a single agent, and the complexity of interactions for heterogeneous combinations requires further research. Third, we also require another validation cohort comprising more distinct clinical factors among samples to confirm our results. Finally, clinical trials are required to verify our results. 

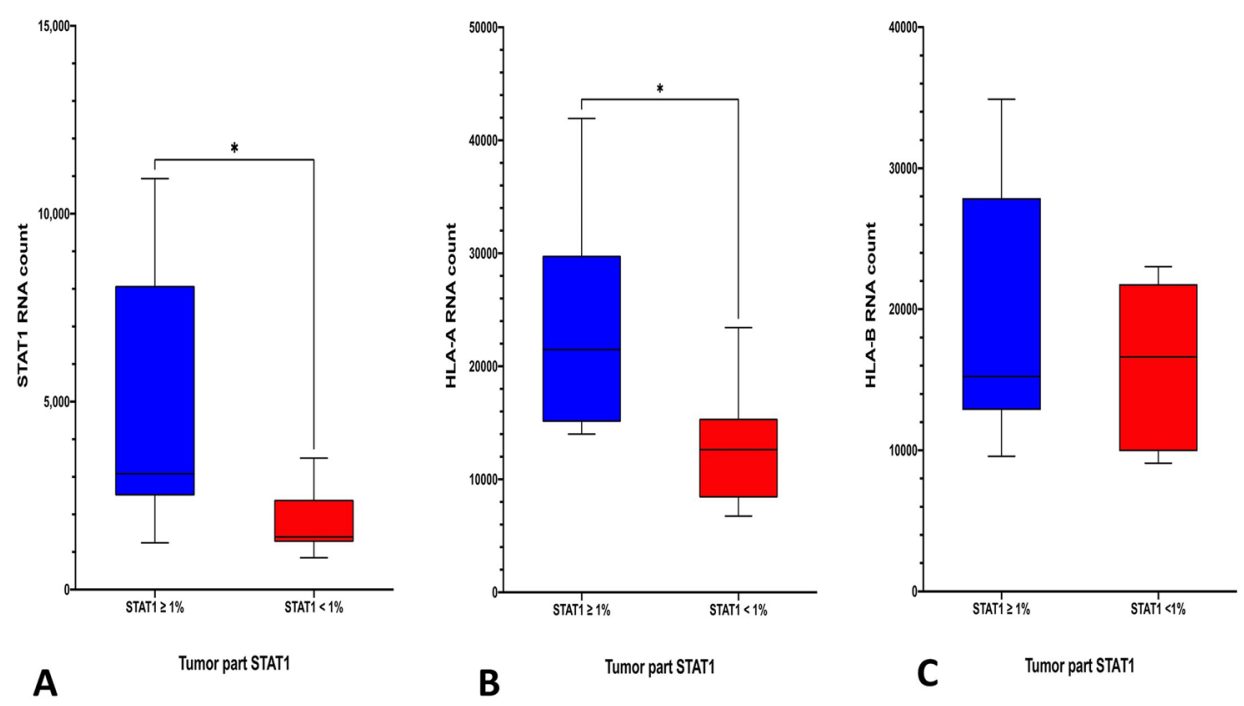

B
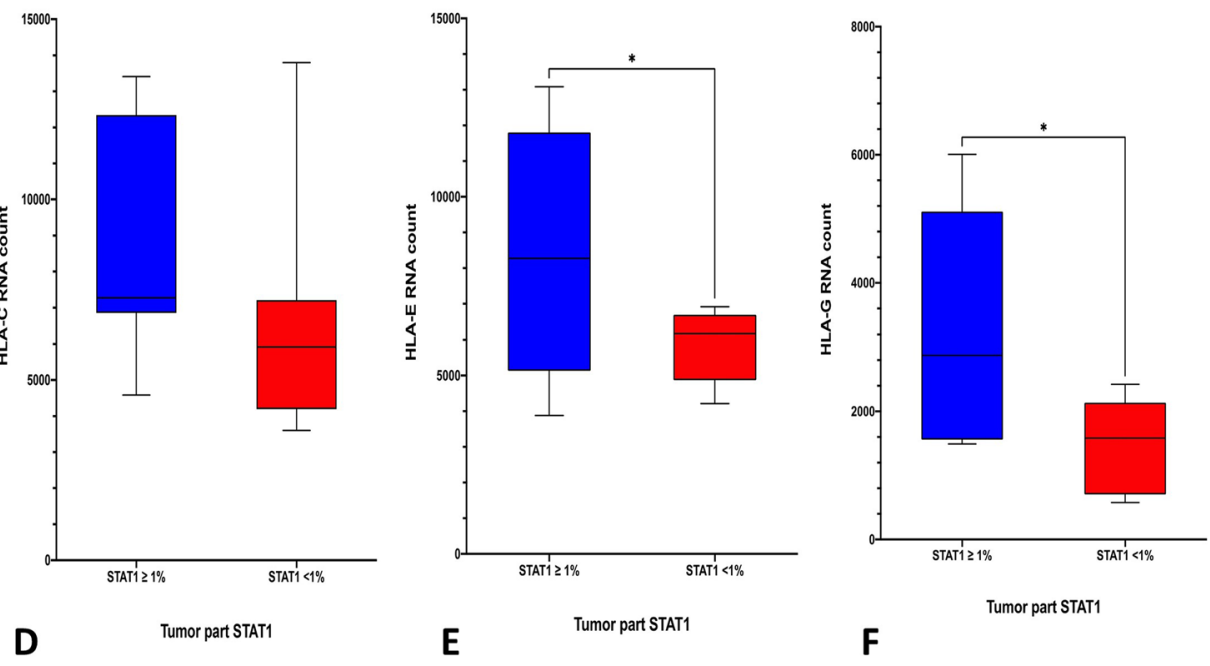

E

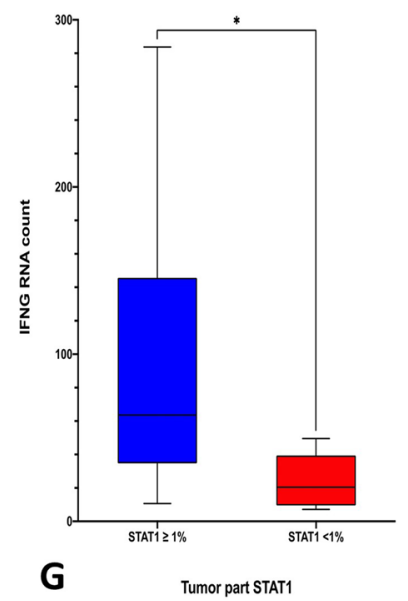

Fig. 5 Counts for mRNA expression in tumor tissue through NanoString analysis for STAT1(A), HLA-A (B), HLA-B (C), HLA-C (D), HLA-E (E), HLA-G (F), and IFN- $\gamma(\mathbf{G})$. Blue bar indicates the results of the high-STAT1 group in the Table 1. Red bar indicates the results of the low-STAT1 group in Table 1. ${ }^{*} p<0.05$ 
Our study successfully established a mechanism underlying immune escape in mCRC through STAT1 downregulation. We also developed an intervention with a proteasome inhibitor to restore the expressions of STAT1 and MHC class I, which is most critical. Our research provides a completely novel rationale for further immunotherapy combinations. This may represent a breakthrough in addressing the challenging treatment landscape of mCRC.

\section{Conclusion}

Our study discovered a new biomarker, the STAT1, for immunotherapy on mCRC treatment. We also provided a potential novel rationale for the addition of bortezomib, a proteasome inhibitor, in new immunotherapy combinations for negative STAT1 mCRC.

\begin{abstract}
Abbreviations
MSI-H: Microsatellite instability-high; mCRC: Metastatic colorectal cancer; 5-FU: 5-Fluorouracil; IFN-ү: Interferon- - ; JAK: Janus kinase; STAT: Signal transducer and activator of transcription; MHC: Major histocompatibility complex; CRC: Colorectal cancer; PD-L1: Programmed cell death-ligand 1; MSS: Microsatellite stable; LLnL: N-acetyl-L-leucyl-L-leucyl-L-norleucinal; HLA: Human leukocyte antigen; PBS: Phosphate-buffered saline; Ig: Immunoglobulin; PE: Phycoerythrin; DSTAT1: Phosphorylated STAT 1; HRP: Horseradish peroxidase; SDS-PAGE: Sodium dodecyl sulfate polyacrylamide gel electrophoresis; DAPI: 4',6-Diamidino-2-phenylindole; IHC: Immunohistochemical; pan-CK: Pan-cytokeratin; IRF1: Interferon regulatory factor-1; TILs: Tumor infiltrating lymphocytes; mRNA: Messenger ribonucleic acid.
\end{abstract}

\section{Supplementary Information}

The online version contains supplementary material available at https://doi. org/10.1186/s12929-021-00769-9.

Additional file 1: Raw data of the PerkinElmer Opal multiplex analysis from 60 samples. The analysis was summarized in Table 1.

\section{Acknowledgements}

Not applicable.

\section{Authors' contributions}

YHL, KHC: contributions to the conception, design of the work, analysis and interpretation of data, draft this manuscript. JHT, YMC: contributions to analysis and interpretation of data. KYC, WLH, CCL, CHK, YTC: contributions to acquisition and interpretation of data. KHY: contributions to the conception, design of the work, interpretation of data. All authors read and approved the final manuscript.

\section{Funding}

This study was supported by the Ministry of Science and Technology, Taiwan (MOST-106-2314-B-002-222-MY3, MOST-109-2634-F-002-043, and MOST-1102634-F-002-044); the Ministry of Education, Taiwan (NTU-109L901403 and NTU-1 10L901404); and National Taiwan University Hospital, Taipei, Taiwan (NTUH.106-N3634).

\section{Availability of data and materials}

We enrolled patients at National Taiwan University Hospital. Detailed information for the processes of PerkinElmer Opal multiplex system is available on the official website at https://www.perkinelmer.com/lab-solutions/resources/ docs/DTS_1-05-40-NR-OPALGUIDELINES_Opal4-7-color_Manual_Kit_Insert. pdf. Quantification of the biomarkers in the tissue sections were performed using the Vectra Polaris Automated Quantitative Pathology Imaging System along with inForm analysis software (PerkinElmer, CLS143455) at our lab. Detailed information and the gene list of NanoString are available on the official website at https://www.nanostring.com/products/gene-expressionpanels/gene-expression-panels-overview/hallmarks-cancer-gene-expressionpanel-collection/pancancer-immune-profiling-panel.

\section{Declarations}

Ethics approval and consent to participate

This study was approved by the Institutional Review Board of National Taiwan University Hospital (IRB No.: NTUH \#201612146RINB), and informed consent was obtained from all patients.

\section{Consent for publication}

Not applicable.

\section{Competing interests}

The authors declare no competing interests in relation to this study.

\section{Author details}

${ }^{1}$ Graduate Institutes of Oncology, National Taiwan University College of Medicine, No 7, Chung-Shan South Rd, Taipei 10002, Taiwan, R.O.C.. ${ }^{2}$ Graduate Institutes of Clinical Medicine, National Taiwan University College of Medicine, Taipei, Taiwan, R.O.C.. ${ }^{3}$ Graduate Institutes of Centers of Genomic and Precision Medicine, National Taiwan University College of Medicine, Taipei, Taiwan, R.O.C.. ${ }^{4}$ Departments of Oncology, National Taiwan University Hospital, Taipei, Taiwan, R.O.C.. ${ }^{5}$ Departments of Pathology, National Taiwan University Hospital, Taipei, Taiwan, R.O.C.. ${ }^{6}$ National Taiwan University Cancer Center, Taipei, Taiwan, R.O.C..

Received: 7 June 2021 Accepted: 20 October 2021

Published online: 10 November 2021

\section{References}

1. Le DT, Uram JN, Wang H, Bartlett BR, Kemberling H, Eyring AD, et al. PD-1 blockade in tumors with mismatch-repair deficiency. N Engl J Med. 2015;372(26):2509-20.

2. Yoshino T, Arnold D, Taniguchi H, Pentheroudakis G, Yamazaki K, Xu RH, et al. Pan-Asian adapted ESMO consensus guidelines for the management of patients with metastatic colorectal cancer: a JSMO-ESMO initiative endorsed by CSCO, KACO, MOS, SSO and TOS. Ann Oncol. 2018;29(1):44-70.

3. Eng C, Kim TW, Bendell J, Argiles G, Tebbutt NC, Di Bartolomeo M, et al, Atezolizumab with or without cobimetinib versus regorafenib in previously treated metastatic colorectal cancer (IMblaze370): a multicentre, open-label, phase 3, randomised, controlled trial. Lancet Oncol. 2019;20(6):849-61.

4. Grothey A, Tabernero J, Arnold D, De Gramont A, Ducreux MP, O'Dwyer PJ, et al. Fluoropyrimidine (FP) + bevacizumab (BEV) + atezolizumab vs $\mathrm{FP} / \mathrm{BEV}$ in BRAFwt metastatic colorectal cancer ( $\mathrm{mCRC}$ ): findings from Cohort 2 of MODUL — a multicentre, randomized trial of biomarker-driven maintenance treatment following first-line induction therapy. Ann Oncol 2018;29(Suppl 8):Abstract LBA19.

5. Singh L, Singh MK, Kenney MC, Jager MJ, Rizvi MA, Meel R, et al. Prognostic significance of PD-1/PD-L1 expression in uveal melanoma: correlation with tumor-infiltrating lymphocytes and clinicopathological parameters. Cancer Immunol Immunother. 2020.

6. Grasso CS, Tsoi J, Onyshchenko M, Abril-Rodriguez G, Ross-Macdonald P, Wind-Rotolo M, et al. Conserved interferon- $\gamma$ signaling drives clinical response to immune checkpoint blockade therapy in melanoma. Cancer Cell. 2020;38(4):500-15.e3.

7. Chang CC, Pirozzi G, Wen SH, Chung IH, Chiu BL, Errico S, et al. Multiple structural and epigenetic defects in the human leukocyte antigen class I antigen presentation pathway in a recurrent metastatic melanoma following immunotherapy. J Biol Chem. 2015;290(44):26562-75.

8. Bukur J, Jasinski S, Seliger B. The role of classical and non-classical HLA class I antigens in human tumors. Semin Cancer Biol. 2012;22(4):350-8. 
9. Groothuis TA, Griekspoor AC, Neijssen JJ, Herberts CA, Neefjes JJ. MHC class I alleles and their exploration of the antigen-processing machinery. Immunol Rev. 2005;207:60-76.

10. Shankaran V, Ikeda H, Bruce AT, White JM, Swanson PE, Old LJ, et al. IFNgamma and lymphocytes prevent primary tumour development and shape tumour immunogenicity. Nature. 2001;410(6832):1107-11.

11. Zhang S, Kohli K, Black RG, Yao L, Spadinger SM, He Q, et al. Systemic interferon- $\gamma$ increases $\mathrm{MHC}$ class i expression and t-cell infiltration in cold tumors: results of a phase 0 clinical trial. Cancer Immunol Res. 2019;7(8):1237-43.

12. Majoros A, Platanitis E, Kernbauer-Hölzl E, Rosebrock F, Müller M, DeckerT. Canonical and non-canonical aspects of JAK-STAT signaling: lessons from interferons for cytokine responses. Front Immunol. 2017:8:29.

13. Gordziel C, Bratsch J, Moriggl R, Knösel T, Friedrich K. Both STAT1 and STAT3 are favourable prognostic determinants in colorectal carcinoma. $\mathrm{Br}$ J Cancer. 2013;109(1):138-46.

14. Nivarthi H, Gordziel C, Themanns M, Kramer N, Eberl M, Rabe B, et al. The ratio of STAT1 to STAT3 expression is a determinant of colorectal cancer growth. Oncotarget. 2016;7(32):51096-106.

15. Leon-Cabrera S, Vázquez-Sandoval A, Molina-Guzman E, DelgadoRamirez Y, Delgado-Buenrostro NL, Callejas BE, et al. Deficiency in STAT1 signaling predisposes gut inflammation and prompts colorectal cancer development. Cancers. 2018;10(9):341.

16. Sakahara M, Okamoto T, Oyanagi J, Takano H, Natsume Y, Yamanaka H, et al. IFN/STAT signaling controls tumorigenesis and the drug response in colorectal cancer. Cancer Sci. 2019;110(4):1293-305.

17. Spano JP, Milano G, Rixe C, Fagard R. JAK/STAT signalling pathway in colorectal cancer: a new biological target with therapeutic implications. Eur J Cancer. 2006:42(16):2668-70.

18. Slattery ML, Lundgreen A, Kadlubar SA, Bondurant KL, Wolff RK. JAK STAT/SOCS-signaling pathway and colon and rectal cancer. Mol Carcinog. 2013;52(2):155-66.

19. Mimura K, Teh JL, Okayama H, Shiraishi K, Kua LF, Koh V, et al. PD-L1 expression is mainly regulated by interferon gamma associated with JAKSTAT pathway in gastric cancer. Cancer Sci. 2018;109(1):43-53.

20. Liang YH, Tsai JH, Cheng YM, Chan KY, Hsu WL, Lee CC, et al. Chemotherapy agents stimulate dendritic cells against human colon cancer cells through upregulation of the transporter associated with antigen processing. Sci Rep. 2021;11(1):9080.

21. Ahmed D, Eide PW, Eilertsen IA, Danielsen SA, Eknæs M, Hektoen M, et al. Epigenetic and genetic features of 24 colon cancer cell lines. Oncogenesis. 2013;2(9):e71.

22. Li Y, Cui K, Zhang Q, Li X, Lin X, Tang Y, et al. FBXL6 degrades phosphorylated p53 to promote tumor growth. Cell Death Differ. 2021;28(7):2112-25.

23. Kwon M, Rubio G, Nolan N, Auteri P, Volmar JA, Adem A, et al. FILIP1L Loss Is a Driver of Aggressive Mucinous Colorectal Adenocarcinoma and Mediates Cytokinesis Defects through PFDN1. Cancer Res. 2021;81(21):5523-39. https://doi.org/10.1158/0008-5472.CAN-21-0897.

24. Ao N, Liu Y, Feng H, Bian X, Li Z, Gu B, et al. Ubiquitin-specific peptidase USP22 negatively regulates the STAT signaling pathway by deubiquitinating SIRT1. Cell Physiol Biochem. 2014;33(6):1863-75.

25. Tundo GR, Sbardella D, Santoro AM, Coletta A, Oddone F, Grasso G, et al. The proteasome as a druggable target with multiple therapeutic potentialities: cutting and non-cutting edges. Pharmacol Ther. 2020;213:107579.

26. Fricker LD. Proteasome inhibitor drugs. Annu Rev Pharmacol Toxicol. 2020;60:457-76.

27. Ten Hoorn S, Trinh A, de Jong J, Koens L, Vermeulen L. Classification of colorectal cancer in molecular subtypes by immunohistochemistry. Methods Mol Biol (Clifton, NJ). 2018;1765:179-91.

28. Bilchik A, Nissan A, Wainberg Z, Shen P, McCarter M, Protic M, et al. Surgical quality and nodal ultrastaging is associated with long-term diseasefree survival in early colorectal cancer: an analysis of 2 international multicenter prospective trials. Ann Surg. 2010;252(3):467-74 (discussion 74-6).

29. Pflügler S, Svinka J, Scharf I, Crncec I, Filipits M, Charoentong P, et al. IDO1(+) Paneth cells promote immune escape of colorectal cancer. Commun Biol. 2020;3(1):252.

30. Yu L, Ye F, Li YY, Zhan YZ, Liu Y, Yan HM, et al. Histone methyltransferase SETDB1 promotes colorectal cancer proliferation through the STAT1CCND1/CDK6 axis. Carcinogenesis. 2020;41(5):678-88.

31. Niu M, Yi M, Dong B, Luo S, Wu K. Upregulation of STAT1-CCL5 axis is a biomarker of colon cancer and promotes the proliferation of colon cancer cells. Ann Transl Med. 2020;8(15):951.

32. Zhao T, Li Y, Zhang J, Zhang B. PD-L1 expression increased by IFN- $\gamma$ via JAK2-STAT1 signaling and predicts a poor survival in colorectal cancer. Oncol Lett. 2020;20(2):1127-34.

33. Kikuchi T, Mimura K, Okayama H, Nakayama Y, Saito K, Yamada L, et al. A subset of patients with MSS/MSI-low-colorectal cancer showed increased CD8(+) TILs together with up-regulated IFN- $\gamma$. Oncol Lett. 2019;18(6):5977-85.

34. Becht E, Giraldo NA, Beuselinck B, Job S, Marisa L, Vano Y, et al. Prognostic and theranostic impact of molecular subtypes and immune classifications in renal cell cancer (RCC) and colorectal cancer (CRC). Oncoimmunology. 2015;4(12):e1049804.

35. Guinney J, Dienstmann R, Wang X, de Reynies A, Schlicker A, Soneson C, et al. The consensus molecular subtypes of colorectal cancer. Nat Med. 2015;21(11):1350-6.

\section{Publisher's Note}

Springer Nature remains neutral with regard to jurisdictional claims in published maps and institutional affiliations.
Ready to submit your research? Choose BMC and benefit from:

- fast, convenient online submission

- thorough peer review by experienced researchers in your field

- rapid publication on acceptance

- support for research data, including large and complex data types

- gold Open Access which fosters wider collaboration and increased citations

- maximum visibility for your research: over 100M website views per year

At BMC, research is always in progress.

Learn more biomedcentral.com/submissions 